\title{
Ein Modell für die neuen Freiwilligenstreitkräfte
}

\author{
Jürgen Groß*
}

\section{Einleitung}

$\mathrm{D}$ ie Aussetzung der allgemeinen Wehrpflicht ist keineswegs nur eine notwendige Konsequenz, die wegen der grundlegenden Veränderung der sicherheitspolitischen Lage in Europa unvermeidlich war (und mit der die Streitkräfte nun eben fertig werden müssen), sondern eine wirkliche Chance für die Bundeswehr. Dies gilt nicht nur in gesellschaftspolitischer Hinsicht, ${ }^{1}$ sondern auch unter militärfunktionalen Gesichtspunkten. Wie die neuen Freiwilligenstreitkräfte mittelfristig aussehen könnten, soll im Folgenden zumindest in groben Umrissen dargestellt werden. Die Überlegungen basieren zu wesentlichen Teilen auf Analysen und Empfehlungen der aus Wissenschaftlerinnen und Wissenschaftlern, Politikern und Militärs zusammengesetzten Kommission „Europäische Sicherheit und Zukunft der Bundeswehr" am Institut für Friedensforschung und Sicherheitspolitik an der Universität Hamburg (IFSH), die schon frühzeitig für eine Abschaffung beziehungsweise Aussetzung der Wehrpflicht auch in Deutschland plädiert hatte. ${ }^{2}$

\section{Sicherheitspolitische Prämissen}

Doch zunächst einmal: Wozu leisten wir uns überhaupt die Bundeswehr? Die in den amtlichen verteidigungspolitischen Grundlagendokumenten genannten „Bedrohungen“, „Gefährdungen“ und „Risiken“3 sind ja zu einem erheblichen Teil nichtmilitärischer Art, das heißt, ihnen ist mit militärischen Mitteln gar nicht beizukommen - völlig unabhängig von der jeweiligen Wehrform. Und selbst in den Fällen, wo dies vielleicht möglich ist, heißt das noch lange nicht, dass ein Einsatz der Bundeswehr auch zulässig, zweckmäßig oder gar notwendig wäre. Mit anderen Worten: Abgesehen vom Verteidigungsauftrag der Streitkräfte gem. Art. 87a GG müssen, bevor deutsche Soldatinnen und Soldaten zum Einsatz kommen, zahlreiche Bedingungen erfüllt sein. Die zentrale Bedingung hat die Kommission am IFSH dabei wie folgt formuliert: „Friedenspolitik ist in allererster Linie eine Politik mit zivilen Mitteln. Der Einsatz militärischer Mittel ist als Maßnahme überhaupt erst dann in Erwägung zu ziehen, wenn sämtliche nichtmilitärischen Instrumente am Ende eines sorgfältigen, gewissenhaften Prüfungs-

* Dr. Jürgen Groß ist Geschäftsführender Vorsitzender der Kommission „Europäische Sicherheit und Zukunft der Bundeswehr“ am Institut für Friedensforschung und Sicherheitspolitik an der Universität Hamburg.

1 Vgl. dazu Jürgen Groß, Die Abschaffung der Wehrpflicht - kein Schaden für die Innere Führung, in: S+F. Sicherheit und Frieden 3/2004, S. 116-118.

2 Vgl. etwa Dieter S. Lutz, Die allgemeine Wehrpflicht: Militärisch überflüssig, verfassungsrechtlich bedenklich, finanziell belastend, in: Reinhard Mutz/Bruno Schoch/Friedhelm Solms (Hrsg.), Friedensgutachten 1997, Münster 1997, S. 185-198 sowie Jürgen Groß (Hrsg.), Europäische Sicherheit und Zukunft der Bundeswehr. Analysen und Empfehlungen der Kommission am IFSH, BadenBaden 2004

3 Bundesministerium der Verteidigung, Verteidigungspolitische Richtlinien, Berlin, 18.5.2011, S. 1. prozesses sowohl auf internationaler als auch auf nationaler Ebene eindeutig als unzureichend angesehen werden müssen und der Einsatz militärischer Mittel mit dem Grundsatz der Verhältnismäßigkeit vereinbar ist. Nichtmilitärische Mittel dürfen auch nicht unter dem Vorwand der möglicherweise mit ihnen einhergehenden hohen finanziellen Aufwendungen von vorneherein als aussichtslos verworfen werden - zumindest nicht, ohne sie zu den mit einem militärischen Einsatz verbundenen Kosten (die in aller Regel weit höher liegen) in Relation zu stellen. “4 Allein unter dieser Prämisse stellt sich überhaupt erst die Frage nach Effizienzsteigerung und struktureller Optimierung der deutschen Streitkräfte. Aber sie stellt sich deshalb nicht weniger dringend.

\section{Auftrag der Bundeswehr}

Nur unter strikter Beachtung der aufgeführten Prämisse also können die Aufgaben der deutschen Streitkräfte künftig wie folgt formuliert werden:

- Mitwirkung an multinationaler Krisenprävention und Konfliktbewältigung,

- Rettung und Evakuierung aus Krisengebieten,

- Verstärkung und Unterstützung der Bündnispartner im Rahmen kollektiver Verteidigung,

- Schutz der Bundesrepublik Deutschland sowie

- Heranziehung zu Hilfeleistungen in Katastrophenfällen im Rahmen der verfügbaren Kapazitäten.

\section{Fähigkeiten der Streitkräfte}

In Ableitung der genannten Aufgaben sollten - einer auch heute noch nicht vollständig umgesetzten Empfehlung der sogenannten „Weizsäcker-Kommission“ aus dem Jahr 2000 folgend - die deutschen Streitkräfte hinsichtlich ihrer Fähigkeiten, Kräfte und Mittel in ihrer Gesamtheit noch konsequenter als bisher auf ihre wahrscheinlichste Aufgabe, die Mitwirkung an multinationaler Krisenprävention und Konfliktbewältigung, ausgerichtet werden, ${ }^{5}$ darüber hinaus in begrenztem Rahmen auch auf die Fähigkeit zur Durchführung von Rettungs- und Evakuierungsmaßnahmen.

Die derzeit bestehende Diskrepanz zwischen den dafür verfügbaren Einsatzkräften und dem Gesamtumfang der Streitkräfte ist daher wesentlich zu verringern. Dieses Ziel sollte vor allem

4 Kommission „Europäische Sicherheit und Zukunft der Bundeswehr“ am IFSH, Auslandseinsätze der Bundeswehr: Viele Bedingungen müssen erfüllt sein, in: S+F. Sicherheit und Frieden 2/2003, S. 153-155, hier S. 153.

5 Vgl. Gemeinsame Sicherheit und Zukunft der Bundeswehr. Bericht der Kommission an die Bundesregierung, Berlin, 23. Mai 2000, S. 48. 
durch eine noch viel weitergehende Reduzierung des Streitkräfteumfangs erreicht werden, als es die aktuellen Planungen des Bundesministeriums der Verteidigung vorsehen. ${ }^{6}$ Demgegenüber sollten die für Auslandseinsätze verfügbaren Kräfte allenfalls moderat erhöht werden. Eine Verdopplung, wie etwa im Bericht der Strukturkommission der Bundeswehr („WeiseKommission“) vorgeschlagen, ${ }^{7}$ mag militärpolitisch begründbar sein, ist jedoch friedenspolitisch das falsche Signal.

Bereits in einem ihrer früheren Papiere hat die Kommission am IFSH darauf hingewiesen, dass nicht jeder militärische Auslandseinsatz auch als Langzeitmission angelegt werden muss, sondern eine solche gleichsam den „Ausnahmefall im Ausnahmefall“ darstellt. ${ }^{8}$ Wenn dieser aber doch eintritt, so ist es - angesichts der vorhandenen Kapazitäten innerhalb der Europäischen Union und der Nordatlantischen Allianz - nicht zwingend notwendig, die Durchhaltefähigkeit der Einsatzkräfte ausschließlich im nationalen Rahmen sicherzustellen. Dabei kann es zweckmäßig sein, standardisierte bi- und multinationale Rotationsverfahren zu entwickeln.

Kapazitäten zur strategischen Verlegefähigkeit sollten ebenfalls nicht im nationalen, sondern im europäischen Rahmen bereitgehalten werden. ${ }^{9}$

Landes- und Bündnisverteidigung bleiben auch in Zukunft originäre Aufgaben der Bundeswehr. Aufgrund der äußerst geringen Eintrittswahrscheinlichkeit beider Fälle rechtfertigen sie jedoch grundsätzlich nicht die Bereitstellung und den Unterhalt eigener Kapazitäten. ${ }^{10}$ Wie im Bericht der „WeizsäckerKommission" festgehalten, sind diese Aufgaben gegebenenfalls mit den primär für Krisenprävention und Konfliktbewältigung bereitgehaltenen Einsatzkräften durchzuführen. ${ }^{11}$ Eine kurzfristige Aufwuchsfähigkeit der Streitkräfte ist in der gegenwärtigen sicherheitspolitischen Lage nicht erforderlich.

\section{Strukturelle Grundlinien}

Unter Fortentwicklung früherer Überlegungen und Empfehlungen der Kommission „Europäische Sicherheit und Zukunft der Bundeswehr"12 sind die immer noch vorhandenen erheblichen Sparpotenziale in der Bundeswehr ohne Rücksicht auf überlebte Traditionen konsequent auszuschöpfen.

Eine zentrale und vordringliche Einzelmaßnahme in diesem Zusammenhang ist die vollständige Verschmelzung der bisher eigenständigen, aufgeblähten Parallelstrukturen der Teilstreit-

6 Vgl. dazu Bundesministerium der Verteidigung, Neuausrichtung der Bundeswehr. Rede des Bundesministers der Verteidigung, Dr. Thomas de Maiziere, am 18.5.2011 in Berlin, S. 15f.

7 Vom Einsatz her denken. Bericht der Strukturkommission der Bundeswehr, Oktober 2010, S. 10.

8 Kommission „Europäische Sicherheit und Zukunft der Bundeswehr“ am IFSH, Am Hindukusch und anderswo - Militäreinsätze ohne Grenzen?, in: S+F. Sicherheit und Frieden 3/2004, S. 152-153, hier S. 152.

9 Vgl. Kommission „Europäische Sicherheit und Zukunft der Bundeswehr“ am IFSH, EUropäische Streitkräfte, in: S+F. Sicherheit und Frieden 1/2011, S. 4144, hier S. 44.

10 Zu den wenigen Ausnahmen zählen bspw. die Alarmrotten im Rahmen des air-policing.

11 Vgl. Gemeinsame Sicherheit, a.a.O.

12 Vgl. insbes. Jürgen Groß, Bundeswehrmodell „200F“, in: S+F. Sicherheit und Frieden 4/2001, S.176-179 sowie Kommission „Europäische Sicherheit und Zukunft der Bundeswehr“ am IFSH, Bundeswehrmodell „2025“, in: S+F. Sicherheit und Frieden 2/2005, S. 107-109. kräfte Heer, Luftwaffe und Marine - ein längst überfälliger Schritt. ${ }^{13}$ Eine Primärgliederung in Teilstreitkräfte stellt seit Langem keine taugliche Grundstruktur für die Funktionalität von Streitkräften mehr dar. Der letzte Zeitpunkt, wo eine solche Unterteilung der bewaffneten Macht einen Sinn ergab, war ungefähr das Jahr 1914. Damals gab es in Deutschland ein Landheer und eine Marine, die in der Tat wenige Berührungspunkte miteinander aufwiesen. Aber schon mit der Entwicklung der Luftstreitkräfte wurden die vormals klaren Trennlinien zwischen einzelnen Teilstreitkräften zunehmend unschärfer. Spätestens mit Beginn des 21. Jahrhunderts sind streitkräftegemeinsame Operationen auch für die Bundeswehr zur Regel geworden. ${ }^{14}$

Es ist unmittelbar einsichtig, dass eine Verschmelzung der Teilstreitkräfte nicht bis hinunter zur Verbands- oder Einheitsebene stattfinden kann. Aber das galt ja auch schon seit jeher analog für Truppenteile mit verschiedenen Aufgaben innerhalb einer Teilstreitkraft, beispielsweise für die verschiedenartigen Truppengattungen im Heer. Teilstreitkräfte als Gliederungskriterium für die höchste militärische Ebene jedenfalls sind heute sachlich kaum noch zu rechtfertigen.

Wie könnte nun stattdessen die Spitzengliederung der Streitkräfte aussehen? Der Generalinspekteur führt die Streitkräfte als unmittelbarer Vorgesetzter. Auf der darunter liegenden Führungsebene erfolgt die Gliederung dann nicht mehr nach Teilstreitkräften beziehungsweise Organisationsbereichen (Zentraler Sanitätsdienst, Streitkräftebasis), sondern nach wirklich funktionalen Kommandos: Einsatzführung, Einsatzvorbereitung, Streitkräfteführung, Ausbildung, Unterstützung. Die bisherigen Inspekteure von Luftwaffe und Marine fungieren künftig nur noch als (herausgehobene) Inspizienten der fliegenden beziehungsweise schwimmenden Verbände, der Inspekteur des Heeres (für den eine analoge Aufgabe wenig sinnvoll wäre) entfällt.

Ebenso entfällt überall die Führungsebene der Division. Die Brigaden sind, dem Einsatzrhythmus entsprechend, den jeweils zuständigen Kommandos direkt unterstellt.

Was heißt: „dem Einsatzrhythmus entsprechend“? Dies soll in schematisierter Form - an einem Beispiel demonstriert werden: Eine bestimmte Brigade ist für einen bestimmten Einsatz vorgesehen. Dazu wird sie zunächst einmal aus dem Verantwortungsbereich des Streitkräfteführungskommandos herausgelöst und dem Einsatzvorbereitungskommando unterstellt. Gleichzeitig wird diese Brigade durch die für ihren Einsatz erforderlichen Unterstützungskräfte (zum Beispiel Kampfhubschrauber, Logistiktruppen, Sanitätstruppen, aber auch Aufklärungs- und Kampfflugzeuge), die bisher allesamt dem Unterstützungskommando unterstellt waren, verstärkt. ${ }^{15}$ In dieser Truppengliederung beginnt sie (unter Nutzung der im Einsatzvorbereitungskommando angesammelten Expertise) mit der spezifischen einsatzbezogenen Ausbildung. Die Aus-

13 Vgl. ebd., S. 178 bzw. 108f.

14 Vgl. dazu etwa die Rede des damaligen Generalinspekteurs der Bundeswehr, General von Kirchbach, am 29.11.1999 auf der 37. Kommandeurtagung in Hamburg.

15 Diese Unterstützungstruppenteile können der Brigade entweder unterstellt oder mit ihr auf Zusammenarbeit angewiesen werden. 
bildungszeit kann bis zu vier Monate betragen; an deren Ende ist die Truppe uneingeschränkt einsatzbereit. ${ }^{16}$

Mit der Verlegung in das Einsatzgebiet wird die Truppe - wie es derzeit bereits der Fall ist - dem Einsatzführungskommando unterstellt. Die Einsatzdauer soll - ebenfalls wie bisher - grundsätzlich vier Monate nicht überschreiten.

Nach Beendigung des Einsatzes tritt die Brigade erneut unter den Befehl des Streitkräfteführungskommandos und nimmt dabei wieder ihre Grundgliederung ein; die für den Einsatz zugeteilten Unterstützungstruppenteile kehren zum Unterstützungskommando zurück. Gleichzeitig endet für einen nicht geringen Teil der Soldatinnen und Soldaten ihre Dienstzeit in der Bundeswehr. ${ }^{17}$ Länger dienendes Personal wird, nach Abgeltung seines Urlaubs, in seiner jeweiligen Funktion beziehungsweise unter Laufbahngesichtspunkten weitergebildet; dies geschieht in Einrichtungen, die in der Zuständigkeit des Ausbildungskommandos liegen. Währenddessen erfolgt in der Brigade mit neuem Personal die Grundausbildung, der sich die Ausbildung im Teileinheits-, Einheits- und Verbandsrahmen anschließt. Neben der personellen findet auch die notwendige materielle Ergänzung statt. Für alle diese Maßnahmen werden eine Einsatznachbereitungs- und eine anschließende Regenerationsphase von insgesamt 16 Monaten veranschlagt, an deren Ende die Brigade wieder grundsätzlich einsatzbereit ist.

Besteht die politische Forderung, dass in einem Einsatzgebiet auf längere Sicht ein deutsches Kontingent im Umfang einer verstärkten Brigade durchhaltefähig präsent sein soll, ergibt sich aus den bisherigen Ausführungen nunmehr ein Bedarf von insgesamt sechs Brigaden. Sollte die Forderung einer gleichzeitigen, durchhaltefähigen Präsenz in zwei Einsatzgebieten gelten, ergibt sich mithin ein Bedarf von zwölf Brigaden. Allerdings könnten die oben angestellten Überlegungen zur Durchhaltefähigkeit im bi- oder multinationalen Rahmen den Bedarf an Einsatzbrigaden wiederum reduzieren. ${ }^{18}$

\section{Personalumfang}

Aufgrund der genannten Anforderungen und strukturellen Grundlagen erscheint mittelfristig eine Reduzierung des deutschen Streitkräfteumfangs auf ca. 130.000 Soldatinnen und Soldaten möglich, davon ungefähr die Hälfte Einsatzkräfte.

Der Personalkörper der Streitkräfte soll sich ausschließlich aus Berufssoldatinnen und Berufssoldaten sowie Soldatinnen und Soldaten auf Zeit zusammensetzen. Um eine möglichst hohe und stetige personelle Fluktuation zwischen Militär und Ge-

16 Einige wenige Truppenteile in der Bundeswehr (z.B. Teile des Kommandos Spezialkräfte) könnten daneben ständig dem Einsatzvorbereitungskommando unterstellt und auf einem (gegenüber den Brigaden des Streitkräfteführungskommandos) erhöhten Bereitschaftsstand gehalten werden, um in kürzester Zeit nach einer spezifischen, ergänzenden Ausbildung in den Einsatz zu gelangen.

17 Siehe dazu unten, Kap. 6.

18 Gerade vor dem Hintergrund der Ausführungen in diesem Kapitel sollte es eine Selbstverständlichkeit sein, das Bundesministerium der Verteidigung organisatorisch gestrafft - unverzüglich und vollständig in Berlin zu konzentrieren. Alle Forderungen nach mehr Effizienz in den Streitkräften bleiben unglaubwürdig, solange ausgerechnet das Ministerium selbst durch seine Aufsplitterung in zwei Standorte in so offensichtlicher Weise gegen diese Forderung verstößt. sellschaft zu gewährleisten, sollte der Anteil der erstgenannten zugunsten der zweiten Kategorie deutlich reduziert werden.

Mit derselben Intention könnte die allgemeine Mindestverpflichtungszeit für Soldatinnen und Soldaten auf Zeit bis auf das militärisch eben noch Vertretbare gesenkt werden, also etwa auf 18 bis 21 Monate. Die Einführung einer zusätzlichen Personalkategorie, also etwa „Freiwilligen Wehrdienst“ Leistende, ist dann militärisch überflüssig.

\section{Schlussbemerkungen}

Die Abschaffung beziehungsweise Aussetzung der Wehrpflicht in Deutschland war seit Langem überfällig, weil sie sicherheitspolitisch nicht mehr zu begründen und damit „ein so tiefer Eingriff in die individuelle Freiheit" ${ }^{19}$ nicht länger zu rechtfertigen war. Derzeit zu beobachtende Anpassungsschwierigkeiten bei der Umstellung auf reine Freiwilligenstreitkräfte müssen nicht überbewertet werden; sie hätten vielleicht noch etwas geringer sein können, wenn viele, die in den letzten eineinhalb Jahrzehnten politische und militärische Verantwortung getragen haben, bereits früher zu der Einsicht gelangt wären, dass die allgemeine Wehrpflicht auf Dauer nicht zu halten sein würde, und ihre Kräfte nicht in Rückzugsgefechten für eine antiquierte Wehrform vergeudet hätten.

Im Übrigen sollte stets vermieden werden, ein und denselben Fehler zweimal zu begehen: Auch im jetzigen Stadium der Bundeswehrtransformation ist die Versuchung wieder groß (in vielerlei Hinsicht, aber ganz besonders in Bezug auf die Teilstreitkräfte), an „Bewährtem“ festzuhalten und bequem gewordene Traditionen nicht preiszugeben - keine guten Voraussetzungen für die notwendige Neuausrichtung der Bundeswehr.

19 Nach dem bekannten Diktum von Roman Herzog, zit. n. Lutz, a.a.O., S. 190 